\title{
Editorial
}

\author{
Beatrice G. Kuhlmann*
}

\section{Topical Issue on Strategy Contributions to Cognitive Aging}

https://doi.org/10.1515/psych-2018-0020

Received June 29, 2019; accepted July 10, 2019

\begin{abstract}
Although some cognitive decline with healthy aging appears inevitable, previous research on strategy instruction and training has repeatedly demonstrated that older adults can substantially improve their cognitive performance through effective strategies. At the same time, age-related changes in strategy repertoire, distribution, execution, and selection have also been documented and, in part, been shown to contribute to the observed age-related deficits in cognitive performance. Authored by researchers from France, Germany, Italy, and the U.S., the nine articles of this Topical Issue on Strategy Contributions to Cognitive Aging provide novel insights on age-related differences (and similarities) in strategies across a variety of cognitive domains (episodic [item, source, event] memory, metamemory, decision making, and numeracy), ranging from new insights on traditional memory-encoding strategies such as self-generation to the discovery of novel strategies involved in event memory, metamemory, and numerosity comparison. Further, a review of event segmentation training and two novel training studies demonstrate much potential for the improvement of older adults' cognitive performance, transferring beyond the trained task-but also identify for whom cognitive strategy training may be less beneficial, necessitating a more intensive or different training approach. All in all, this Topical Issue provides a comprehensive picture of age-related changes in cognitive strategies and means to improve older adults' strategic approach to cognitive tasks.
\end{abstract}

Keywords: cognitive aging, cognitive strategies, metamemory, training, transfer

Presumably, we all wish to age successfully, including maintaining cognitive functioning not merely at a sufficient level for independent living but at a sufficient level to entertain meaningful social relationships (e.g., by remembering names) and to bring personal joy (e.g., in reminiscing). Striving for this personally, I have always been excited by research showing that observed age-related differences in cognitive performance are in part overestimated because they are confounded with age-related changes in other factors, with one primary factor being age-related changes in strategic task approach (see Lemaire, 2010, for a comprehensive review). At the same time, it has baffled me that, according to this research, "old dogs" will not always perform the tricks they have learned, as for example repeatedly shown in the research by my dissertation mentor (Touron, 2015) and also evident in the lack of transfer effects of most training studies despite successful improvements on the trained tasks (Rebok, Carlson, \& Langbaum, 2007). I view the study of age-related changes in cognitive strategies as central to successful cognitive aging because strategies afford the three key ingredients of successful aging (Baltes \& Baltes, 1993): selection (e.g., memory encoding strategies focusing attention on relevant information), optimization (e.g., strategy training), and

Article note: This article is a part of Topical Issue "Strategy Contributions to Cognitive Aging” edited by Beatrice G. Kuhlmann. The author thanks Dayna R. Touron for valuable comments on a draft of this text.

*Corresponding author: Beatrice G. Kuhlmann, Department of Psychology, School of Social Sciences, University of Mannheim, Schloss EO 240, 68131 Mannheim, Germany, E-mail: kuhlmann@psychologie.uni-mannheim.de 
compensation (e.g., switching to novel strategies to maintain performance). By discovering which cognitive strategies are most effective in older adults and how we can teach and support older adults to apply these strategies to a variety of cognitive tasks, we can ultimately help older adults to age successfully.

I was thus very pleased with the opportunity to guest edit this Topical Issue on Strategy Contributions to Cognitive Aging. Now being completed, I think this Topical Issue compiles an insightful collection of novel research and perspectives on the possibilities as well as limitations of cognitive improvements through strategies in older adulthood. As summarized in further detail below, the studies of this Topical Issue demonstrate aging-spared memory benefits of encoding strategies that even extend to memory types that are profoundly impaired in older age (i.e., associative and source memory; McCurdy, Leach, \& Leshikar, 2019). Some of the contributed studies uncover novel cognitive strategies used by older adults in metamemory monitoring (Parlett-Pelleriti, Lin, Jones, Linstead, \& Jaeggi, 2019) and numerosity estimation (Roquet \& Lemaire, 2019), and one presents a successful novel strategy training of older adults' decisionmaking skills (Rosi, Vecchi, \& Cavallini, 2019). Furthermore, this and another (Cavallini, Bottiroli, Dunlosky, Ambiel, Lux, \& Hertzog, 2019) training study not only find their older trainees to improve on the trained tasks but observe transfer gains to non-trained tasks. Together, the nine contributions demonstrate that high cognitive performance remains well possible in older age with the help of cognitive strategies.

In the following, I will briefly summarize the nine contributions of this Topical Issue. I will start with the studies providing new insights on memory-encoding strategies, the traditional focus of cognitive strategy research. Next, I will highlight the contributing studies demonstrating the role of strategies for understanding age differences in various other cognitive domains beyond memory. Finally, I will discuss the novel insights from the contributed training studies, which in particular increases our understanding of how to foster transfer gains in older trainees.

\section{New Insights on Strategic Memory Encoding in Older Age}

Traditionally, much research on cognitive strategies has focused on encoding strategies in episodic memory tasks (Craik \& Tulving, 1975; Richardson, 1998) and such mnemonic strategies have also been the focus of the early investigations of strategy contributions to cognitive aging (Kausler, 1994). Keeping with this tradition, four of the nine manuscripts (and one additional training study discussed later) in this Topical Issue examine memory-encoding strategies in older adults.

Expanding on the well-established memory benefit of self-referential encoding (Rogers, Kuiper, \& Kirker, 1977), the study contributed by Lipitz, Liui, and Gutchess (2019) investigated the relationship between self-referential encoding and mind-wandering in younger and older adults. They found the memory benefits of self-referential encoding to be spared from aging. Notably, they also observed that selffocus during encoding decreased mind-wandering, which is detrimental to memory performance, in both age groups. Interestingly, older adults overall mind-wandered less than younger adults during any type of encoding task, extending this well-established age difference from the mind-wandering literature (e.g., Giambra, 1989; Jackson \& Balota, 2012) to memory encoding. In sum, the study by Lipitz et al. demonstrates that self-referential memory processing is fully spared from aging, with older adults being well able to focus on the self-referential encoding task.

The study contributed by McCurdy et al. (2019) also examines a strategy-benefit to memory that is spared from aging: the generation effect (Slamecka \& Graf, 1978). Extending previous research that focused primarily on item memory, they show that generation also benefits older adults' associative and source memory despite the much larger age-related deficits in these types of memory as compared to their item memory deficits (Chalfonte \& Johnson, 1996; Naveh-Benjamin, 2000; Old \& Naveh-Benjamin, 2008). However, source-memory improvements with generation appear somewhat limited in older adults who did not draw any additional source-memory benefits from less-constrained self-generation (vs. more constrained generation in the form of unscrambling an anagram) whereas younger adults reached the highest level of source memory when generation was the least constrained. Nonetheless, the finding of improvement in older adults' source memory with any type of generation task (self-generation, unscrambling an anagram) is exciting given the large age-related impairments in this type of memory. 
Older adults' pronounced source-memory deficit is also the focus of the study contributed by Mitchell and Hill (2019). They observed similar relationships between encoding focus and source memory in younger and older adults, suggesting that older adults' source-memory deficit does not arise from them attending to different aspects during spontaneous encoding than younger adults. Notable about their approach is the inclusion of measures of subjective experience during encoding, which corroborate that older adults were as able to follow the encoding-focus instructions as younger adults. Together with the study by McCurdy et al. (2019), these results imply that there are pronounced age-related deficits in source memory beyond any strategy differences but, fortunately, that older adults can nonetheless improve their source memory through encoding strategies (see also Kuhlmann \& Touron, 2012).

Moving from the encoding of isolated item+source episodes, the review contributed by McGatlin, Newberry, and Bailey (2019) argues for the potential of a recently-discovered encoding strategy to improve memory encoding of complex events in older adults: event segmentation. They point to first empirical evidence that despite age-related impairments in spontaneous event segmentation, older adults can use their preserved semantic knowledge to compensate for these segmentation deficits and can further be successfully trained to segment even unfamiliar scenes.

\section{Beyond Memory Encoding: Aging and Strategies in Other Cognitive Domains}

Four contributions in this Topical Issue examine older adults' strategy abilities in cognitive domains beyond memory encoding. Two contributions address older adults' metamemory judgments. Using data from an impressive field study on eyewitness memory, Martschuk, Sporer, and Sauerland (2019) show that confidence in the identification-response at test becomes less predictive of identification accuracy in eyewitnesses aged 40 and older. Specifically, older eyewitnesses were as confident in their identification responses as younger eyewitnesses despite the typical age-related declines in identification accuracy, resulting in the older eyewitnesses' overconfidence. Future research should determine why older eyewitnesses are increasingly overconfident in their identification decisions. Perhaps older adults misperceive cues diagnostic for identification accuracy and/or switch to less diagnostic cues to make their confidence judgment. Martschuk et al.'s additional analyses suggest that a misperception of decision time in older adults may be one contributing factor (see also Hertzog, Touron, \& Hines, 2007). The contribution by Parlett-Pelleriti et al. (2019) uses an innovative machine-learning-based methodological approach to identify and compare metamemory monitoring strategies in younger and older adults. Like Martschuk et al. they observed overconfidence in older adults' metamemory judgments, which in this study were assessed at the time of learning (vs. at test in Martschuk et al.) in the form of placing bets on one's upcoming memory performance (cf., McGillivray \& Castel, 2011). The hierarchical clustering analysis identified two strategy clusters pertaining to both younger and older adults with no age differences in strategy distribution. Unfortunately, older adults' overconfidence did not improve across the five rounds of the memory-betting task (but see McGillivray \& Castel, 2011). Building on these two studies, hopefully future research can identify strategies to decrease older adults' metamemory overconfidence, which is robustly found (see Castel, Middlebrooks, \& McGillivray, 2016, for a review).

Moving to the domain of numeracy, the contribution by Roquet and Lemaire (2019) demonstrates younger and older adults' use of a broad repertoire of strategies for making numerosity comparisons. Their findings document both age-related strategy differences (e.g., in strategy preferences) but also strategy similarities (e.g., in the number of strategies used by individuals). Future research on age-related differences in numerosity comparisons should thus attend to and account for these strategic factors.

Finally, thecontribution by Rosi etal.(2019) presents anovel metacognitive strategy-training that substantially improves older adults' decision making compared to an active control group. In addition to promoting an analytical mode of thinking, their training borrows from a metacognitive learner-oriented approach that helps foster transfer effects of strategy training by guiding learners in how to think about implementing the newly learned strategies in other tasks (Bottiroli, Cavallini, Dunlosky, Vecchi, \& Hertzog, 2013). Indeed, the training group in the study by Rosi et al. also improved on non-trained decision-making tasks. 


\section{Advances in Cognitive Strategy Training and Transfer in Older Adults}

This Topical Issue features two strategy-training studies with older trainees (Cavallini et al., 2019; Rosi et al., 2019). Excitingly, both training interventions yielded large improvements in older adults' cognitive performance (memory and decision making, respectively). In addition to the strategy-training component, both of these trainings followed the above-described metacognitive learner-oriented approach invented by Bottiroli et al. (2013). This approach was developed in response to the longstanding issue that, despite large improvements on the trained tasks, training effects notoriously show little to no transfer to nontrained tasks (see Rebok et al., 2007, for a review). The metacognitive learner-oriented approach addresses this issue by devoting part of the training to teaching the trainees the metacognitive steps necessary for adapting the trained strategies to a novel task. Fortunately, both studies indeed observed transfer of training improvements to non-trained tasks, replicating Bottiroli et al.'s initial positive evaluation results of this approach. Notably, whereas Cavallini et al. used the same memory-strategy training as Bottiroli et al., Rosi et al. successfully adapted the metacognitive learner-oriented approach to the training of decisionmaking strategies.

In addition, the contributed study by Cavallini et al. (2019) expands upon the previous research on the learner-oriented training approach for memory strategies by examining individual differences in training and transfer gains. Interestingly, they observed that transfer gains, but not initial training gains on the trained task, depended on the older trainees' working memory capacity, processing speed, and verbal knowledge. Future research should explore how the metacognitive transfer-training component may be more easily followed and implemented by older adults with reduced cognitive resources, for example by altering the presentation format and allowing older adults to write down intermediate steps of the adaptation questions (Bottiroli, Dunlosky, Guerini, Cavallini, \& Hertzog, 2010; Kuhlmann \& Touron, 2016). Such task modifications may also foster transfer by improving strategy implementation on the trained tasks; in the study by Cavallini et al., gains on the trained task significantly predicted transfer gains beyond the aforementioned cognitive resource measures and other control variables.

All in all, the contributed training studies show that older adults can be successfully trained to transfer cognitive strategies to novel tasks, finally overcoming the tenacious lack of transfer effects in strategytraining studies. Further in line with this, the review contributed by McGatlin et al. (2019) points to first empirical evidence that older adults' event segmentation ability can be successfully trained. Because gains from segmentation training were observed on unfamiliar events, it seems likely that these gains will broadly transfer to novel events.

\section{Conclusion}

Together, the nine contributions to this Topical Issue convincingly demonstrate that older adults greatly benefit from strategies in a variety of cognitive domains (episodic [item, source, event] memory, metamemory, decision making, and numeracy). The contributing studies provide new insights on wellestablished strategies (e.g., self-referential processing and the generation effect), discover novel strategies (e.g., for numerosity comparisons), and advance our understanding of a novel strategy-training approach to afford transfer of training gains. I am convinced that these studies will provide a good foundation on which future research can build, for example to devise strategies to counteract metamemory overconfidence in older adults and to foster transfer of strategy training for various cognitive-strategy training approaches. 


\section{References}

Baltes, P. B., \& Baltes, M. M. (1993). Psychological perspectives on successful aging: The model of selective optimization with compensation. In P. B. Baltes \& M. M. Baltes (Eds.), Successful aging: Perspectives from the behavioral sciences (pp. 1-34). New York, NY, US: Cambridge Univ Press.

Bottiroli, S., Cavallini, E., Dunlosky, J., Vecchi, T., \& Hertzog, C. (2013). The importance of training strategy adaptation: a learner-oriented approach for improving older adults' memory and transfer. Journal of Experimental Psychology: Applied, 19, 205-218. doi:10.1037/a0034078

Bottiroli, S., Dunlosky, J., Guerini, K., Cavallini, E., \& Hertzog, C. (2010). Does task affordance moderate age-related deficits in strategy production? Aging, Neuropsychology, and Cognition, 17, 591-602. doi:10.1080/13825585.2010.481356

Castel, A. D., Middlebrooks, C. D., \& McGillivray, S. (2016). Monitoring Memory in Old Age: Impaired, Spared, and Aware. In J. Dunlosky \& S. U. Tauber (Eds), The Oxford Handbook of Metamemory (pp. 519-534). doi:10.1093/ oxfordhb/9780199336746.013.3

Cavallini, E., Bottiroli, S., Dunlosky, J., Ambiel, E., Lux, A., \& Hertzog, C. (2019). Strategy-adaptation memory training: predictors of older adults' training gains. Open Psychology, 1, 255-272. doi:10.1515/psych-2018-0017

Chalfonte, B. L., \& Johnson, M. K. (1996). Feature memory and binding in young and older adults. Memory \& Cognition, 24, 403-416. doi:10.3758/BF03200930

Craik, F. I. M., \& Tulving, E. (1975). Depth of processing and the retention of words in episodic memory. Journal of Experimental Psychology: General, 104, 268-294. doi:10.1037/0096-3445.104.3.268

Giambra, L. M. (1989). Task-unrelated-thought frequency as a function of age: A laboratory study. Psychology and Aging, 4, 136-143. doi:10.1037/0882-7974.4.2.136

Hertzog, C., Touron, D. R., \& Hines, J. C. (2007). Does a time-monitoring deficit influence older adults' delayed retrieval shift during skill acquisition? Psychology and Aging, 22, 607-624. doi:10.1037/0882-7974.22.3.607

Jackson, J. D., \& Balota, D. A. (2012). Mind-wandering in younger and older adults: Converging evidence from the sustained attention to response task and reading for comprehension. Psychology and Aging, 27, 106-119. doi:10.1037/a0023933

Kausler, D. H. (1994). Learning and memory in normal aging. San Diego, CA, USA: Academic Press.

Kuhlmann, B. G., \& Touron, D. R. (2012). Mediator-based encoding strategies in source monitoring in young and older adults. Journal of Experimental Psychology: Learning, Memory, and Cognition, 38, 1352-1364. doi:10.1037/a0027863

Kuhlmann, B. G., \& Touron, D. R. (2016). Aging and memory improvement through semantic clustering: The role of list-presentation format. Psychology and Aging, 31, 771-785. doi:10.1037/pag0000117

Lemaire, P. (2010). Cognitive strategy variations during aging. Current Directions in Psychological Science, 19, 363-369. doi:10.1177/0963721410390354

Lipitz, S. R., Liu, X., \& Gutchess, A. (2019). Self-referential memory encoding and mind- wandering in younger and older adults. Open Psychology, 1, 58-68. doi:10.1515/psych-2018-0005

Martschuk, N., Sporer, S. L., \& Sauerland, M. (2019). Confidence of older eyewitnesses: Is it diagnostic of identification accuracy? Open Psychology, 1, 132-151. doi:10.1515/psych-2018-0010

McCurdy, M. P., Leach, R. C., \& Leshikar, E. D. (2019). Fewer constraints enhance the generation effect for source memory in younger, but not older adults. Open Psychology, 1, 168-184. doi:10.1515/psych-2018-0012

McGatlin, K. C., Newberry, K. M., \& Bailey, H. R. (2019). Temporal chunking makes life's events more memorable. Open Psychology, 1, 94-105. doi:10.1515/psych-2018-0007

McGillivray, S., \& Castel, A. D. (2011). Betting on memory leads to metacognitive improvement by younger and older adults. Psychology and Aging, 26, 137-142. doi:10.1037/a0022681

Mitchell, K. J., \& Hill, E. M. (2019). The impact of focusing on different features during encoding on young and older adults' source memory. Open Psychology, 1, 106-118. doi:10.1515/psych-2018-0008

Naveh-Benjamin, M. (2000). Adult age differences in memory performance: Tests of an associative deficit hypothesis. Journal of Experimental Psychology: Learning, Memory, and Cognition, 26, 1170-1187. doi:10.1037//0278-7393.26.5.1170

Old, S. R., \& Naveh-Benjamin, M. (2008). Differential effects of age on item and associative measures of memory: A meta-analysis. Psychology and Aging, 23, 104-118. doi:10.1037/0882-7974.23.1.104

Parlett-Pelleriti, C., Lin, G. C., Jones, M. R., Linstead, E., \& Jaeggi, S. M. (2019). Exploring age-related metamemory differences using modified brier scores and hierarchical clustering. Open Psychology, 1, 215-238. doi:10.1515/psych-2018-0015

Rebok, G. W., Carlson, M. C., \& Langbaum, J. B. S. (2007). Training and maintaining memory abilities in healthy older adults: Traditional and novel approaches. The Journals of Gerontology: Series B, 62, 53-61. doi:10.1093/geronb/62.special_ issue_1.53

Richardson, J. T. E. (1998). The availability and effectiveness of reported mediators in associative learning: A historical review and an experimental investigation. Psychonomic Bulletin \& Review, 5, 597-614. doi:10.3758/BF03208837

Rogers, T. B., Kuiper, N. A., \& Kirker, W. S. (1977). Self-reference and the encoding of personal information. Journal of Personality and Social Psychology, 35, 677-688. doi:10.1037/0022-3514.35.9.677

Roquet, A., \& Lemaire, P. (2019). Strategy variability in numerosity comparison task: a study in young and older adults. Open Psychology, 1, 152-167. doi:10.1515/psych-2018-0011 
Rosi, A., Vecchi, T., \& Cavallini, E. (2019). Metacognitive-strategy training promotes decision-making ability in older adults. Open Psychology, 1, 200-214. doi:10.1515/psych-2018-0014

Slamecka, N. J., \& Graf, P. (1978). The generation effect: Delineation of a phenomenon. Journal of Experimental Psychology: Human Learning \& Memory, 4, 592-604. doi:10.1037/0278-7393.4.6.592

Touron, D. R. (2015). Memory avoidance by older adults: When “old dogs” won’t perform their “new tricks." Current Directions in Psychological Science, 24, 170-176. doi:10.1177/0963721414563730 